GLASNIK MATEMATIČKI

Vol. 39(59)(2004), $185-198$

\title{
SOME QUESTIONS OF EQUIVARIANT MOVABILITY
}

\author{
P.S. Gevorgyan \\ Moscow State University, Russia
}

\begin{abstract}
In this article some questions of equivariant movability, connected with the substitution of the acting group $G$ on closed subgroup $H$ and with transitions to spaces of $H$-orbits and $H$-fixed points spaces, are investigated. In a special case, the characterization of equivariantly movable $G$-spaces is given.
\end{abstract}

\section{INTRODUCTION}

This paper is devoted to equivariant movability of $G$-spaces, i.e., topological spaces endowed with an action of a given compact group $G$.

More precisely, in $\S 3$ we define the notion of equivariant movability or $G$-movability and we prove several theorems, including the following ones. If $X$ is $p$-paracompact and $H \subseteq G$ is a closed subgroup, then $G$-movability of $X$ implies its $H$-movability ( $\S 3$, Theorem 3.3). $G$-movability of $X$ also implies movability of the space $X[H]$ of $H$-fixed points in $X(\S 4$, Theorem 4.1). In particular, equivariant movability of a $G$-space $X$ implies ordinary movability of the topological space $X(\S 3$, Corollary 3.5). We construct a non-trivial example which shows, that the converse, in general, is not true, even if we take for $G$ the cyclic group $Z_{2}$ of order $2(\S 5$, Example 5.1). If $X$ is a metrizable $G$-movable space and $H$ is a closed normal subgroup of $G$, then the space $\left.X\right|_{H}$ of its $H$-orbits is also $G$-movable ( $\S 6$, Theorem 6.1). In the case $H=G$ we obtain that $G$-movability of a metrizable $G$-space implies ordinary movability of the orbit space $\left.X\right|_{G}(\S 6$, Corollary 6.2). The last assertion, in general, is not invertible ( $\S 6$, Example 6.3). However, if $X$ is metrizable, $G$ is a compact Lie group and the action of $G$ on $X$ is free, then $X$ is $G$-movable if and only if the orbit space $\left.X\right|_{G}$ is movable (§ 7, Theorem 7.2). Examples $6.3(\S 6)$ and

2000 Mathematics Subject Classification. 55P55, 54C56.

Key words and phrases. Equivariant shape theory, equivariant movability. 
$3(\S 8)$ show that in the last theorem the assumption that the group $G$ is a Lie group and the assumption that the action is free cannot be omitted.

Some of the above listed results with an outline of proof were given in [9].

Let us denote the category of all topological spaces and continuous maps by Top, the category of all metrizable spaces and continuous maps by $M$ and the category of all $p$-paracompact spaces and continuous maps by $P$. Always in this article it is assumed that all topological spaces are $p$-paracompact spaces and the group $G$ is compact.

The author is extremely grateful to the referee for his helpful remarks and comments.

The reader is referred to the books by K. Borsuk [4] and by S. Mardešić and J. Segal [15] for general information about shape theory and to the book by G. Bredon [5] for introduction to compact transformation groups.

\section{BASIC NOTIONS AND CONVENTIONS CONCERNING EQUIVARIANT TOPOLOGY}

Let $G$ be a topological group. A topological space $X$ is called a $G$-space if there is a continuous map $\theta: G \times X \rightarrow X$ of the direct product $G \times X$ into $X, \theta(g, x)=g x$, such that

$$
\text { 1) } g(h x)=(g h) x ; \quad 2) \quad e x=x \text {, }
$$

for all $g, h \in G, x \in X$; here $e$ is the unity of $G$. Such a (continuous) map $\theta: G \times X \rightarrow X$ is called an (continuous) action of the group $G$ on the topological space $X$. An evident example is the so called trivial action of $G$ on $X: g x=x$, for all $g \in G, x \in X$. Another example is the action of the group $G$ on itself, defined by $(g, x) \rightarrow g x$ for all $g \in G, x \in G$.

If $X$ and $Y$ are $G$-spaces, then so is $X \times Y$, where $g(x, y)=(g x, g y)$, $g \in G,(x, y) \in X \times Y$.

A subset $A$ of a $G$ space $X$ is called invariant provided $g \in G, a \in A$ implies $g a \in A$. It is evident, that an invariant subset of a $G$ space is itself a $G$ space. If $A$ is an invariant subset of a $G$ space $X$, then every neighborhood of $A$ contains an open invariant neighborhood of $A$ (see [17], Proposition 1.1.14).

Let $X$ be any $G$-space and let $H$ be a closed and normal subgroup of the group $G$. The set $H x=\{h x ; h \in H\}$ is called the $H$-orbit of the point $x \in X$. Clearly the $H$-orbits of any two points in $X$ are either equal or disjoint, in other words $X$ is partitioned by its $H$-orbits. We denote the set of all $H$-orbits of the $G$-space $X$ by $\left.X\right|_{H}$. The set $\left.X\right|_{H}$ endowed with the quotient topology is called the $H$-orbit space of $X$. There is a continuous action of the group $G$ on the space $\left.X\right|_{H}$ defined by the formula $g H x=H g x, g \in G, x \in X$. So, $\left.X\right|_{H}$ is a $G$-space. In case $H=G$ the $G$-orbit of the point $x \in X$ is called the orbit of the point $x$ and the $G$-orbit space is called the orbit space of the $G$-space $X$. 
We denote by $X[H]$ the subspace of fixed points of $H$ on $X$, or the $H$-fixed point subspace of the $G$-space $X$. Let us recall that $X[H]=\{x \in X ; h x=x$, for any $h \in H\}$.

The set $G_{x}=\{g \in G ; g(x)=x\}$ is a closed subgroup of the group $G$, for every $x \in X . G_{x}$ is called the stationary subgroup (or stabilizer) at the point $x$. The action of the group $G$ on $X$ (or the $G$-space $X$ ) is called free if the stationary subgroup $G_{x}$ is trivial, for every $x \in X$. It is clear that $G_{g x}=g G_{x} g^{-1}$, i.e., the stationary subgroups at any two points of the same orbit are conjugate. The orbits $G x$ and $G y$ of points $x$ and $y$, respectively, are said to have the same type if the stationary subgroups $G_{x}$ and $G_{y}$ are conjugate.

Let $X, Y$ be $G$-spaces. A (continuous) map $f: X \rightarrow Y$ is called a $G$-map, or an equivariant map, if $f(g x)=g f(x)$ for every $g \in G, x \in X$. Note that the identity map $i: X \rightarrow X$ is equivariant and the composition of equivariant maps is equivariant. Therefore, all $G$-spaces and equivariant maps form a category. Let us denote the category of all topological $G$-spaces and equivariant maps by $T o p_{G}$, the category of all metrizable $G$-spaces and equivariant maps by $M_{G}$ and the category of all $p$-paracompact $G$-spaces and equivariant maps by $P_{G}$.

Let $Z$ be a $G$-space and let $Y \subseteq Z$ be an invariant subset. A $G$-retraction of $Z$ to $Y$ is a $G$-map $r: Z \rightarrow Y$ such that $\left.r\right|_{Y}=1_{Y}$.

Let $K_{G}$ be class of $G$-spaces. A $G$-space $Y$ is called a $G$-absolute neighborhood retract for the class $K_{G}$ or a $G-A N R\left(K_{G}\right)(G$-absolute retract for the class $K_{G}$ or a $G-A R\left(K_{G}\right)$ ), provided $Y \in K_{G}$ and whenever $Y$ is a closed invariant subset of a $G$-space $Z \in K_{G}$, then there exist an invariant neighborhood $U$ of $Y$ and a $G$-retraction $r: U \rightarrow Y$ (there exists a $G$-retraction $r: Z \rightarrow Y)$.

A $G$-space $Y$ is called a $G$-absolute neighborhood extensor for the class $K_{G}$ or a $G-A N E\left(K_{G}\right)\left(G\right.$-absolute extensor for the class $K_{G}$ or a $G-$ $\left.A E\left(K_{G}\right)\right)$, provided for any $G$-space $X \in K_{G}$ and any closed invariant subset $A \subseteq X$, every equivariant map $f: A \rightarrow Y$ admits an equivariant extension $\tilde{f}: \bar{U} \rightarrow Y$, where $U$ is an invariant neighborhood of $A$ in $X(\tilde{f}: X \rightarrow Y)$.

\section{Movability AND EQUivariant MOVABILITy}

The important shape invariant, called movability, was originally introduced by K. Borsuk [2] for metric compacta. Mardešić and Segal [14] generalized the notion of movability to compacta using the $A N R$-system approach. Kozlowski and Segal in [11] gave a categorical description of this property which applied to arbitrary topological spaces.

Following Mardešić and Segal [14], let us define the notion of equivariant movability or $G$-movability : 
Definition 3.1. An inverse $G$-system $\underline{X}=\left\{X_{\alpha}, p_{\alpha \alpha^{\prime}}, A\right\}$ where each $X_{\alpha}$, $\alpha \in A$, is a $G$-space and every $p_{\alpha \alpha^{\prime}}: X_{\alpha^{\prime}} \rightarrow X_{\alpha}, \alpha \leqslant \alpha^{\prime}$, is a G-homotopy class, is called equivariantly movable or $G$-movable if for every $\alpha \in A$, there exists an $\alpha^{\prime} \in A, \alpha^{\prime} \geqslant \alpha$ such that for all $\alpha^{\prime \prime} \in A, \alpha^{\prime \prime} \geqslant \alpha$ there exists a $G$-homotopy class $r^{\alpha^{\prime} \alpha^{\prime \prime}}: X_{\alpha^{\prime}} \rightarrow X_{\alpha^{\prime \prime}}$ such that

$$
p_{\alpha \alpha^{\prime \prime}} \circ r^{\alpha^{\prime} \alpha^{\prime \prime}}=p_{\alpha \alpha^{\prime}} \text {. }
$$

It is known (see [1], Theorem 2) that every $G$-space $X$ admits a $G-A N R$ expansion in the sense of Mardešić (see [15], I, § 2.1), which is the same as saying that there is an inverse $G-A N R$-system ( $G$-system consisting of $G-A N R$ 's) $\underline{X}=\left\{X_{\alpha}, p_{\alpha \alpha^{\prime}}, A\right\}$ associated with $X$ in the sense of Morita [16].

Definition 3.2. A G-space $X$ is called equivariantly movable or $G$ movable if there is an equivariantly movable inverse $G-A N R$-system $\underline{X}=$ $\left\{X_{\alpha}, p_{\alpha \alpha^{\prime}}, A\right\}$ associated with $X$.

Note that the last definition of equivariant movability coincides with the notion of ordinary movability if $G=\{e\}$ is the trivial group.

Let $X$ be an equivariantly movable $G$-space. The evident question arises: does movability of the space $X$ follows from its equivariant movability? The following, more general theorem gives a positive answer (Corollary 3.5) to the above question.

TheOREM 3.3. Let $H$ be a closed subgroup of a group $G$. Every $G$-movable $G$-space is $H$-movable.

To prove this theorem the next result is important.

THEOREM 3.4. Let $H$ be a closed subgroup of a group $G$. Every $G-$ $A R\left(P_{G}\right)\left(G-A N R\left(P_{G}\right)\right)$-space is an $H-A R\left(P_{H}\right)\left(H-A N R\left(P_{H}\right)\right)$-space.

Proof. According to a theorem of de Vries ([7], Theorm 4.4), it is sufficient to show that if $X$ is a $p$-paracompact $H$-space, then the twisted product $G \times_{H} X$ is also $p$-paracompact. Indeed, since $X$ is $p$-paracompact and $G$ is compact, $G \times X$ is $p$-paracompact. Therefore, the twisted product $G \times{ }_{H} X$ is $p$-paracompact.

Proof of Theorem 3.3. Let $X$ be any equivariantly movable $G$-space. With respect to the theorem of Smirnov ([18], Theorem 1.3), there is a closed and equivariant embedding of the $G$-space $X$ to some $G-A R\left(P_{G}\right)$-space $Y$. Let us consider all open $G$-invariant neighborhoods of type $F_{\sigma}$ of the $G$-space $X$ in $Y$. By a result of R. Palais ([17], Proposition 1.1.14), these neighborhoods form a cofinal family in the set of all open neighborhoods of $X$ in $Y$, in particular, in the set of all open and $H$-invariant neighborhoods of the $H$-space $X$ in the $H$-space $Y$, which, by Theorem 3.3 is an $H-A R\left(P_{H}\right)$ space. Hence, from the $G$-movability of the above mentioned family follows 
its $H$-movability, i.e. from the $G$-movability of the $G$-space $X$ follows the $H$-movability of the $H$-space $X$.

From Theorem 3.3 we obtain the following corollary if we consider the trivial subgroup $H=\{e\}$ of the group $G$.

COROllary 3.5. Every equivariantly movable $G$-space $X$ is movable.

The converse, in general, is not true, even if one takes for $G$ the cyclic group $Z_{2}$ of order 2 (see Example 5.1).

\section{Movability of the $H$-FiXed Point SpaCe}

TheOREm 4.1. Let $H$ be a closed subgroup of a group $G$. If a $G$-space $X$ is equivariantly movable, then the $H$-fixed point space $X[H]$ is movable.

The proof requires the use of the following theorem.

Theorem 4.2. Let $H$ be a closed subgroup of a group $G$. Let $X$ be a $G-A R\left(P_{G}\right)\left(G-A N R\left(P_{G}\right)\right)$ - space. Then the $H$-fixed point space $X[H]$ is an $A R(P)(A N R(P))$-space.

Proof. Let $X$ be a $G-A R\left(P_{G}\right)\left(G-A N R\left(P_{G}\right)\right)$-space. By Theorem 3.4, it is sufficient to prove the theorem in the case $H=G$. I.e., we must prove that $X[G]$ is $A R(P)$-space. By a theorem of Smirnov ([18], Theorem 1.3), we can consider $X$ as a closed $G$-subspace of a $G-A R\left(P_{G}\right)$-space $C(G, V) \times \prod D_{\lambda}$ where $V$ is a normed vector space and thus an $A E(M)$-space, $C(G, V)$ is the space of continuous maps from $G$ to $V$ with the compact-open topology and with the action $\left(g^{\prime} f\right)(g)=f\left(g g^{\prime}\right), g, g^{\prime} \in G, f \in C(G, V)$ of the group $G$ and $D_{\lambda}$ is a closed ball of a finite-dimensional Euclidean space $E_{\lambda}$ with the orthogonal action of the group $G$.

First, let us prove that the set $\left(C(G, V) \times \prod D_{\lambda}\right)[G]$ of all fixed points of the $G$-space $C(G, V) \times \prod D_{\lambda}$ is an $A R(P)$-space. The spaces $C(G, V)$ and $E_{\lambda}$ are normed spaces. Since the actions of the group $G$ on $C(G, V)$ and $E_{\lambda}$ are linear, the sets $C(G, V)[G]$ and $E_{\lambda}[G]$ will be closed convex sets of locally convex spaces $C(G, V)$ and $E_{\lambda}$, respectively. Therefore, by a wellknown theorem of Kuratowski and Dugundji [3], $C(G, V)$ and $E_{\lambda}$ are absolute retracts for metrizable spaces. By a theorem of Lisica [12], they are also absolute retracts for $p$-paracompact spaces. For a closed ball $D_{\lambda} \subset E_{\lambda}$ the last conclusion is true since the set $D_{\lambda}[G]=D_{\lambda} \bigcap E_{\lambda}[G]$ is closed and convex in $E_{\lambda}$.

Since the group $G$ acts on the product $C(G, V) \times \prod D_{\lambda}$ coordinate-wise,

$$
\left(C(G, V) \times \prod D_{\lambda}\right)[G]=C(G, V)[G] \times\left(\prod D_{\lambda}\right)[G] .
$$

Hence, $\left(C(G, V) \times \prod D_{\lambda}\right)[G]$ is an $A R(P)$-space, because it is a product of two $A R(P)$-spaces. 
Now let us prove that $X[G]$ is an $A R(P)$-space. Since $X$ is a $G-A R\left(P_{G}\right)$ space, it is a $G$-retract of the product $C(G, V) \times \prod D_{\lambda}$. Therefore, $X[G]$ is a retract of the $A R(P)$-space $\left(C(G, V) \times \prod D_{\lambda}\right)[G]$, hence, it is an $A R(P)$-space.

The absolute neighborhood retract case is proved similarly.

Proof of Theorem 4.1. Let $X$ be a $G$-movable space. By Theorem 3.3 , it is sufficient to prove the theorem in the case $H=G$. So, we must prove movability of the space $X[G]$ of all $G$-fixed points. We consider the $G$-space $X$ as a closed and $G$-invariant space of some $G-A R\left(P_{G}\right)$-space $Y$ ([18], Theorem 1.3). The family of all open, $G$-invariant $F_{\sigma}$-type neighborhoods $U_{\alpha}$ of the $G$-space $X$ in $Y$, is cofinal in the set of all open neighborhoods of $X$ in $Y$ ([17], Proposition 1.1.14). It consists of $G-A N R\left(P_{G}\right)$-spaces. The intersections $U_{\alpha} \cap Y[G]=U_{\alpha}[G]$ are $A N R(P)$-spaces (Theorem 4.2). They form a cofinal family of neighborhoods of the space $X[G]$ in $Y[G]$. Indeed, for any neighborhood $U$ of the set $X[G]$ in $Y[G]$ there is a neighborhood $V$ of the set $X[G]$ in $Y$ such that $V \cap Y[G]=U$. Then the set $W=(Y \backslash Y[G]) \cup V$ is a neighborhood of the set $X$ in $Y$, moreover, $W \cap Y[G]=U$. There is an $\alpha$ such that $U_{\alpha} \subset W$ and therefore $U_{\alpha}[G] \subset U$. So the family of neighborhoods $U_{\alpha}[G]$ is cofinal.

Since $X$ is $G$-movable, for every $U_{\alpha}$ there is a neighborhood $U_{\alpha^{\prime}} \subset U_{\alpha}$ such that, for any other neighborhood $U_{\alpha^{\prime \prime}} \subset U_{\alpha^{\prime}}$, there exists a $G$-equivariant homotopy $F: U_{\alpha^{\prime}} \times I \rightarrow U_{\alpha}$ such that $F(y, 0)=y$ and $F(y, 1) \in U_{\alpha^{\prime \prime}}$, for any $y \in U_{\alpha^{\prime}}$. It is not difficult to verify that the homotopy $F[G]: U_{\alpha^{\prime}}[G] \times I \rightarrow$ $U_{\alpha}[G]$, induced by $F$, satisfies the condition of movability of $X[G]$.

\section{Example of a movable, But nOt Equivariantly movable SPACE}

EXAmPle 5.1. We will use the idea of S. Mardešić [13]. Let us consider the unit circle $S=\{z \in C ;|z|=1\}$. Let us denote $B=[S \times\{1\}] \cup[\{1\} \times S]$. $B$ is the wedge of two copies of the unit circle $S$ with base point $\{1\}$. Let us define a continuous map $f: B \rightarrow B$ by the formulas:

$$
\begin{gathered}
f(z, 1)= \begin{cases}\left(z^{4}, 1\right), & 0 \leqslant \arg (z) \leqslant \frac{\pi}{2} \\
\left(1, z^{4}\right), & \frac{\pi}{2} \leqslant \arg (z) \leqslant \pi \\
\left(z^{-4}, 1\right), & \pi \leqslant \arg (z) \leqslant \frac{3 \pi}{2} \\
\left(1, z^{-4}\right), & \frac{3 \pi}{2} \leqslant \arg (z) \leqslant 2 \pi\end{cases} \\
f(1, t)= \begin{cases}\left(t^{-4}, 1\right), & 0 \leqslant \arg (t) \leqslant \frac{\pi}{2} \\
\left(1, t^{-4}\right), & \frac{\pi}{2} \leqslant \arg (t) \leqslant \pi \\
\left(t^{4}, 1\right), & \pi \leqslant \arg (t) \leqslant \frac{3 \pi}{2} \\
\left(1, t^{4}\right), & \frac{3 \pi}{2} \leqslant \arg (t) \leqslant 2 \pi\end{cases}
\end{gathered}
$$


for every $z$ and $t$ from $S$. Let us consider the $A N R$-sequences

$$
B \stackrel{f}{\longleftarrow} B \stackrel{f}{\longleftarrow} B \stackrel{f}{\longleftarrow} \cdots
$$

and

$$
\Sigma B \stackrel{\Sigma f}{\longleftarrow} \Sigma B \stackrel{\Sigma f}{\longleftarrow} \Sigma B \stackrel{\Sigma f}{\longleftarrow} \cdots
$$

where $\Sigma$ is the operation of suspension. Let us denote

$$
P=\lim _{\longleftarrow}\{B, f\} \text {. }
$$

Then

$$
\Sigma P=\lim _{\longleftarrow}\{\Sigma B, \Sigma f\} .
$$

Let us define an action of the group $Z_{2}=\{e, g\}$ on $\Sigma B$ by the formulas

$$
e[x, t]=[x, t] ; \quad g[x, t]=[x,-t] .
$$

for every $[x, t] \in \Sigma B,-1 \leqslant t \leqslant 1$. It induces an action on $\Sigma P$.

Proposition 5.2. The space $\Sigma P$ has trivial shape, but it is not $Z_{2}$ movable.

Proof. The triviality of shape of the space $\Sigma P$ is proved by the method of Mardešić [13]. Let us prove that the space $\Sigma P$ is not $Z_{2}$-movable. Consider the set $\Sigma P\left[Z_{2}\right]$ of all fixed-points of $Z_{2}$-space $\Sigma P$. It is obvious that $\Sigma P\left[Z_{2}\right]=$ $P$. Hence, by Theorem 4.1, it is sufficient to prove the following proposition.

Proposition 5.3. The space $P$ is not movable.

Proof. Since the movability of an inverse system remains unchanged under the action of a functor, it is sufficient to prove non-movability of the inverse sequence of groups

$$
\pi_{1}(B) \stackrel{f_{*}}{\longleftarrow} \pi_{1}(B) \stackrel{f_{*}}{\longleftarrow} \pi_{1}(B) \stackrel{f_{*}}{\longleftarrow} \cdots,
$$

where $\pi_{1}(B)$ is the fundamental group of the space $B$ and $f_{*}$ is the homomorphism induced by the mapping $f: B \rightarrow B$.

It is known that for sequences of groups movability implies the following condition of Mittag-Leffler, abbreviated as $M L$ ([15], p. 166, Corollary 4):

The inverse system $\left\{G_{\alpha}, p_{\alpha \alpha^{\prime}}, A\right\}$ of the pro - GROUP category is said to be $M L$ provided for every $\alpha \in A$, there exist $\alpha^{\prime} \in A, \alpha^{\prime} \geqslant \alpha$, such that $p_{\alpha \alpha^{\prime}}\left(G_{\alpha^{\prime}}\right)=p_{\alpha \alpha^{\prime \prime}}\left(G_{\alpha^{\prime \prime}}\right)$, for any $\alpha^{\prime \prime} \in A, \alpha^{\prime \prime} \geqslant \alpha$.

Thus, it sufficient to prove that the sequence (1) does not satisfy condition $M L$. Let us observe that $\pi_{1}(B)$ is a free group with two generators $a$ and $b$, and $f_{*}$ is the homomorphism defined by the formulas

$$
f_{*}(a)=a b a^{-1} b^{-1}, \quad f_{*}(b)=a^{-1} b^{-1} a b .
$$


$f_{*}$ is a monomorphism, because $f_{*}(a) \neq f_{*}(b)$, but not an epimorphism, because, for example, $f_{*}(x) \neq a$, for all $x \in \pi_{1}(B)$. Hence, for any natural $m$ and $n, I m f_{*}^{m} \varsubsetneqq I m f_{*}^{n}$ only if $m>n$. It means that the inverse sequence (1) does not satisfy condition $M L$.

\section{Movability of the ORBIT SPACE}

TheOrem 6.1. Let $X$ be a metrizable $G$-space. If $X$ is $G$-movable then for any closed and normal subgroup $H$ of the group $G$, the $H$-orbit space $\left.X\right|_{H}$ is also G-movable.

Proof. Without losing generality one may suppose that $X$ is a closed $G$-invariant subset of some $G-A R\left(M_{G}\right)$-space $Y\left([18]\right.$, Theorem 1.1). $\left.X\right|_{H}$ is a closed $G$-invariant subset of $\left.Y\right|_{H}([5]$, Theorem 3.1).

Let $\left\{X_{\alpha}, \alpha \in A\right\}$ be the family of all $G$-invariant neighborhoods of $X$ in $Y$. Let us consider the family $\left\{\left.X_{\alpha}\right|_{H}, \alpha \in A\right\}$, where each $\left.X_{\alpha}\right|_{H} \in G-A N R\left(M_{G}\right)$ and is a $G$-invariant neighborhood of $\left.X\right|_{H}$ in $\left.Y\right|_{H}$. Let us prove that the family $\left\{\left.X_{\alpha}\right|_{H}, \alpha \in A\right\}$ is cofinal in the family of all neighborhoods of $\left.X\right|_{H}$ in $\left.Y\right|_{H}$. Let $U$ be an arbitrary neighborhood of $\left.X\right|_{H}$ in $\left.Y\right|_{H}$. By a theorem of Palais ([17], Proposition 1.1.14), there exists a $G$-invariant neighborhood $\left.V \supset X\right|_{H}$ laying in $U$. Let us denote $\tilde{V}=(p r)^{-1}(V)$, where $p r:\left.Y \rightarrow Y\right|_{H}$ is the $H$-orbit projection. It is evident that $\tilde{V}$ is a $G$-invariant neighborhood of the space $X$ in $Y$ and $V=\left.\tilde{V}\right|_{H}$. So in any neighborhood of the space $\left.X\right|_{H}$ in $\left.Y\right|_{H}$, there is a neighborhood of type $\left.X_{\alpha}\right|_{H}$, where $X_{\alpha}$ is a $G$-invariant neighborhood of $X$ in $Y$.

Now let us prove the $G$-movability of the space $\left.X\right|_{H}$. Let $X$ be $G$-movable. It means that the inverse system $\left\{X_{\alpha}, i_{\alpha \alpha^{\prime}}, A\right\}$ is $G$-movable. We must prove that the induced inverse system $\left\{\left.X_{\alpha}\right|_{H},\left.i_{\alpha \alpha^{\prime}}\right|_{H}, A\right\}$ is $G$-movable. Let $\alpha \in A$ be any index. By the $G$-movability of the inverse system $\left\{X_{\alpha}, i_{\alpha \alpha^{\prime}}, A\right\}$, there is $\alpha^{\prime} \in A, \alpha^{\prime}>\alpha$, such that for any other index $\alpha^{\prime \prime} \in A, \alpha^{\prime \prime}>\alpha$, there exists a $G$-mapping $r^{\alpha^{\prime} \alpha^{\prime \prime}}: X_{\alpha^{\prime}} \rightarrow X_{\alpha^{\prime \prime}}$, which makes the following diagram $G$-homotopy commutative

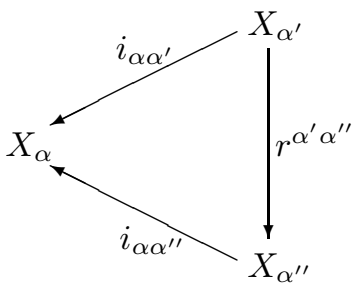

DiAgram 1. 
It turns out that, for given $\alpha \in A$, the obtained index $\alpha^{\prime} \in A, \alpha^{\prime}>\alpha$, also satisfies the condition of $G$-movability of the inverse system

$$
\left\{\left.X_{\alpha}\right|_{H},\left.i_{\alpha \alpha^{\prime}}\right|_{H}, A\right\} \text {. }
$$

This is obvious, because the $G$-homotopy commutativity of Diagram 1 implies the $G$-homotopy commutativity of the following diagram

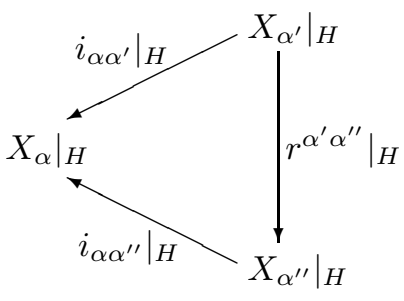

\section{DIAGRAM 2.}

where $\left.r^{\alpha^{\prime} \alpha^{\prime \prime}}\right|_{H}:\left.\left.X_{\alpha^{\prime}}\right|_{H} \rightarrow X_{\alpha^{\prime \prime}}\right|_{H}$ is induced by the mapping $r^{\alpha^{\prime} \alpha^{\prime \prime}}$. So, the $G$-movability of the space $\left.X\right|_{H}$ is proved.

Corollary 6.2. Let $X$ be a metrizable $G$-space. If $X$ is $G$-movable, then the orbit space $\left.X\right|_{G}$ is movable.

Proof. In the case $H=G$ from the last theorem we obtain that the orbit space $\left.X\right|_{G}$ with the trivial action of the group $G$ is $G$-movable. Therefore, it will be movable by Corollary 3.5.

Corollary 6.2 in general is not invertible:

ExAmple 6.3. Let $\Sigma$ be a solenoid. It is known ([4], Theorem 13.5) that $\Sigma$ is a non-movable compact metrizable Abelian group. By Corollary 3.5, the solenoid $\Sigma$ with the natural group action is not $\Sigma$-movable although the orbit space $\left.\Sigma\right|_{\Sigma}$ as a one-point set is movable.

The converse of Corollary 6.2 is true if the group $G$ is a Lie group and the action is free (see Theorem 7.2).

\section{Equivariant movability of a fReE $G$-SPACE}

Theorem 7.1. Let $G$ be a compact Lie group and let $Y$ be a metrizable $G-A R\left(M_{G}\right)$-space. Suppose that a closed invariant subset $X$ of $Y$ has an invariant neighborhood whose orbits have the same type. If the orbit space $\left.X\right|_{G}$ is movable, then $X$ is equivariantly movable.

Proof. The orbit space $\left.X\right|_{G}$ is closed in $\left.Y\right|_{G}$, which is a $G-A R(M)$ space. Let $U$ be an arbitrary invariant neighborhood of $X$ in $Y$. By the assumption of the theorem, it follows that there exists a cofinal family of neighborhoods of $X$ in $Y$, whose orbits have the same type. Therefore, one 
may suppose that all orbits of the neighborhood $U$ have the same type. The orbit set $\left.U\right|_{G}$ will be a neighborhood of $\left.X\right|_{G}$ in $\left.Y\right|_{G}$. From the movability of $\left.X\right|_{G}$ it follows that, for the neighborhood $\left.U\right|_{G}$, there is a neighborhood $\tilde{V}$ of the space $\left.X\right|_{G}$ in $\left.Y\right|_{G}$, which lies in the neighborhood $\left.U\right|_{G}$ and contracts to any preassigned neighborhood of the space $\left.X\right|_{G}$.

Let us denote $V=(p r)^{-1}(\tilde{V})$, where $p r:\left.Y \rightarrow Y\right|_{G}$ is the orbit projection. It is evident that $V$ is an invariant neighborhood of the space $X$ lying in $U$. Let us prove that $V$ contracts in $U$ to any preassigned invariant neighborhood of $X$. Let $W$ be any invariant neighborhood of $X$ in $Y$. We must prove the existence of an equivariant homotopy $F: V \times I \rightarrow U$, which satisfies the condition

$$
F(x, 0)=x, \quad F(x, 1) \in W,
$$

for any $x \in V$. Since $\left.W\right|_{G}$ is a neighborhood of the space $\left.X\right|_{G}$ in $\left.Y\right|_{G}$, there is a homotopy $\tilde{F}:\left.V\right|_{G} \times\left. I \rightarrow U\right|_{G}$ such that

$$
F(\tilde{x}, 0)=\tilde{x},\left.\quad \tilde{F}(\tilde{x}, 1) \in W\right|_{G},
$$

for any $\left.\tilde{x} \in V\right|_{G}$. The homotopy $\tilde{F}:\left.V\right|_{G} \times\left. I \rightarrow U\right|_{G}$ preserves the $G$ orbit structure, because $V \subset U$ and all orbits of $U$ have the same types (see Diagram 3).

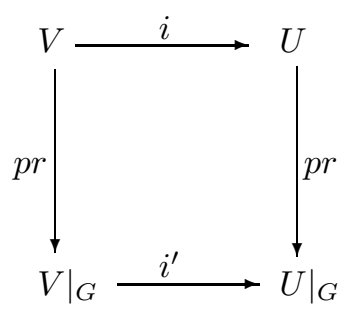

DiAgRAM 3.

By the covering homotopy theorem of Palais ([17], Theorem 2.4.1), there is an equivariant homotopy $F: V \times I \rightarrow U$, which covers the homotopy $\tilde{F}$ and satisfies $F(x, 0)=i(x)=x$. That is, the following diagram is commutative (Diagram 4).

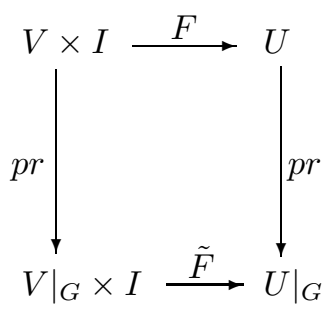

Diagram 4. 
$F: V \times I \rightarrow U$ is the designed equivariant homotopy. It only remains to verify that $F(x, 1) \in W$. But this immediately follows from (2) and the commutativity of Diagram 4.

TheOREm 7.2. Let $G$ be a compact Lie group. A metrizable free $G$-space $X$ is equivariantly movable if and only if the orbit space $\left.X\right|_{G}$ is movable.

Proof. The necessity in a more general case was proved in Corollary 6.2. Let us prove the sufficiency. Let the orbit space $\left.X\right|_{G}$ be movable. One can consider the $G$-space $X$ as a closed and invariant subset of some $G-A R\left(M_{G}\right)$ space $Y$. Let $P \subset X$ be any orbit. From the existence of slices it follows that around $P$ there is such an invariant neighborhood $U(P)$ in $Y$ that type $Q \geqslant$ type $P$, for any orbit $Q$ from $U(P)$ ([5], Corollary 5.5). Since the action of the group $G$ on $X$ is free, type $Q=$ type $P=$ type $G$, for any orbit $Q$ lying in $U(P)$. Let us denote $V=\cup\left\{U(P) ;\left.P \in X\right|_{G}\right\}$. It is evident that $V$ is an invariant neighborhood of the space $X$ in $Y$ and that all of its orbits have the same type. Then, by Theorem 7.1, $X$ is equivariantly movable.

Example 6.3 shows that the assumption that $G$ is a Lie group is essential in the above theorem. The Example 8.1 which follows shows that the condition of freeness of the action of the group $G$ is also essential in the above theorem.

\section{ExAmple of A NON-FREe nOt $Z_{2}$-MOVABle SPACE With A MOVABle ORBIT SPACE}

EXAmPle 8.1. Let us consider the space $P=\lim \{B, f\}$ constructed in Example 5.1. Let us define an action of the group $Z_{2}=\{e, g\}$ on the space $B$ by the formulas

$$
\begin{aligned}
& e(z, 1)=(z, 1) \\
& e(1, t)=(1, t) \\
& g(z, 1)=\left(1, z^{-1}\right) \\
& g(1, t)=\left(t^{-1}, 1\right),
\end{aligned}
$$

for any $z$ and $t$ from $S$. $B$ is a $Z_{2}-A N R\left(M_{Z_{2}}\right)$ space with the fixed-point $b_{0}=(1,1)$.

Proposition 8.2. The mapping $f: B \rightarrow B$, defined by formulas (3), is equivariant.

Proof. It is necessary to prove the following two equalities:

$$
\begin{aligned}
& f(g(z, 1))=g(f(z, 1)) \\
& f(g(1, t))=g(f(1, t)),
\end{aligned}
$$

for any $z$ and $t$ from $S$. Let us prove the first one. Consider the following cases: 


$$
\begin{aligned}
& \text { Case 1. } 0 \leqslant \arg z \leqslant \frac{\pi}{2} \quad \Leftrightarrow \quad \frac{3 \pi}{2} \leqslant \arg z^{-1} \leqslant 2 \pi . \\
& \text { Then } f(g(z, 1))=f\left(1, z^{-1}\right)=\left(1, z^{-4}\right)=g\left(z^{4}, 1\right)=g f(z, 1) \text {. } \\
& \text { Case 2. } \frac{\pi}{2} \leqslant \arg z \leqslant \pi \quad \Leftrightarrow \quad \pi \leqslant \arg z^{-1} \leqslant \frac{3 \pi}{2} . \\
& \text { Then } f(g(z, 1))=f\left(1, z^{-1}\right)=\left(z^{-4}, 1\right)=g\left(1, z^{4}\right)=g f(z, 1) \text {. } \\
& \text { Case 3. } \pi \leqslant \arg z \leqslant \frac{3 \pi}{2} \Leftrightarrow \quad \Leftrightarrow \quad \frac{\pi}{2} \leqslant \arg z^{-1} \leqslant \pi . \\
& \text { Then } f(g(z, 1))=f\left(1, z^{-1}\right)=\left(1, z^{4}\right)=g\left(z^{-4}, 1\right)=g f(z, 1) \text {. } \\
& \text { Case 4. } \frac{3 \pi}{2} \leqslant \arg z \leqslant 2 \pi \Leftrightarrow 0 \leqslant \arg z^{-1} \leqslant \frac{\pi}{2} . \\
& \text { Then } f(g(z, 1))=f\left(1, z^{-1}\right)=\left(z^{4}, 1\right)=g\left(1, z^{-4}\right)=g f(z, 1) .
\end{aligned}
$$

The second equality of (4) is proved in a similar way.

Proposition 8.3. $P$ is a connected, compact, metrizable and equivariantly non-movable $Z_{2}$-space which is free at all points except at the only fixed point $\left(b_{0}, b_{0}, \ldots\right)$ and $\operatorname{sh}\left(\left.P\right|_{Z_{2}}\right)=0$.

Proof. $P$ is a $Z_{2}$-space because it is an inverse limit of $Z_{2}-A N R\left(M_{Z_{2}}\right)$ spaces $B$ and $f$ is an equivariant mapping. The uniqueness of the fixed point is evident. The connectedness, compactness and metrizability follows from the properties of inverse systems ([8], Theorem 6.1.20, Corollary 4.2.5). The non $Z_{2}$-movability follows from Proposition 5.3 and Corollary 3.5 .

Let us prove that $\operatorname{sh}\left(\left.P\right|_{Z_{2}}\right)=0$ and thus the orbit space $\left.P\right|_{Z_{2}}$ is movable.

Let $X=\lim _{\lfloor}\left\{\left.B\right|_{Z_{2}},\left.f\right|_{Z_{2}}\right\} . \quad X$ is equimorphic to the orbit space $\left.P\right|_{Z_{2}}$. Indeed, let us define a mapping $h:\left.X \rightarrow P\right|_{Z_{2}}$ in the following way:

$$
h\left(\left(\left[x_{1}\right],\left[x_{2}\right], \ldots\right)\right)=\left[\left(x_{1}, x_{2}, \ldots\right)\right]
$$

where $\left(\left[x_{1}\right],\left[x_{2}\right], \ldots\right) \in X$, and $x_{1}, x_{2}, \ldots$ are selected from the classes $\left[x_{1}\right],\left[x_{2}\right], \ldots$ in such way that $\left(x_{1}, x_{2}, \ldots\right) \in P$ or what is the same $f\left(x_{n+1}\right)=$ $x_{n}$, for any $n=1,2, \ldots$. Let us prove that the mapping $h$ is defined correctly. Let $\tilde{x}_{1}, \tilde{x}_{2}, \ldots$ be some other representatives of the classes $\left[x_{1}\right],\left[x_{2}\right], \ldots$, respectively, satisfying the conditions $f\left(\tilde{x}_{n+1}\right)=\tilde{x}_{n}$ for any $n \in N$. Since each class $\left[x_{n}\right]$ has two representatives: $x_{n}$ and $g x_{n}$, where $g \in Z_{2}=\{e, g\}$, either $\tilde{x}_{n}=g x_{n}$ or $\tilde{x}_{n}=x_{n}$. But it is obvious that, if for some $n_{0} \in N, \tilde{x}_{n_{0}}=g x_{n_{0}}$, then, for any $n \in N, \tilde{x}_{n}=g x_{n}$, because $f$ is equivariant. Thus, in the case of another choice of the representatives of the classes $\left[x_{1}\right],\left[x_{2}\right], \ldots$, we have

$$
\begin{aligned}
& h\left(\left(\left[x_{1}\right],\left[x_{2}\right], \ldots\right)\right)=\left[\left(\tilde{x}_{1}, \tilde{x}_{2}, \ldots\right)\right]=\left[\left(g x_{1}, g x_{2}, \ldots\right)\right]= \\
&= {\left[g\left(x_{1}, x_{2}, \ldots\right)\right]=\left[\left(x_{1}, x_{2}, \ldots\right)\right] . }
\end{aligned}
$$

However, $h$ is a continuous bijection and thus, it is a homeomorphism ([8], Theorem 3.1.13).

Consequently,

$$
\left.P\right|_{Z_{2}}=\lim _{\longleftarrow}\left\{\left.B\right|_{Z_{2}},\left.f\right|_{Z_{2}}\right\},
$$


where $\left.B\right|_{Z_{2}} \cong S$ and the mapping $\bar{f}=\left.f\right|_{Z_{2}}: S \rightarrow S$ is defined by the formulas:

$$
\bar{f}(z)= \begin{cases}z^{4}, & 0 \leqslant \arg (z) \leqslant \frac{\pi}{2} \\ z^{-4}, & \frac{\pi}{2} \leqslant \arg (z) \leqslant \frac{3 \pi}{2} \\ z^{4}, & \frac{3 \pi}{2} \leqslant \arg (z) \leqslant 2 \pi\end{cases}
$$

for any $z \in S$. Thus, we conclude that the orbit space $\left.P\right|_{Z_{2}}$ is a limit of the inverse sequence

$$
S \stackrel{\bar{f}}{\longleftarrow} S \stackrel{\bar{f}}{\longleftarrow} S \stackrel{\bar{f}}{\longleftarrow} \cdots
$$

By formula (5), the mapping $\bar{f}$ induces a homomorphism $\bar{f}_{*}: \pi_{1}(S) \rightarrow \pi_{1}(S)$, which acts as follows:

$$
\bar{f}_{*}(a)=a a^{-1} a^{-1} a,
$$

where $a \in \pi_{1}(S) \cong Z$ is the generator of the group $Z$. From the above formula, it follows that $\bar{f}_{*}$ is the null-homomorphism and thus, $\operatorname{deg} \bar{f}=0$. For any $k=1,2, \cdots, \bar{f}_{*}^{k}$ is also a null-homomorphism and thus, $\operatorname{deg} \bar{f}^{k}=0$. Therefore, by the classical Hopf theorem ([10], Section 2.8, Theorem $H^{n}$ ) all $\bar{f}^{k}: S \rightarrow S$ are null-homotopic and $\operatorname{sh}\left(\left.P\right|_{Z_{2}}\right)=0$.

\section{REFERENCES}

[1] S.A. Antonian and S. Mardešić, Equivariant shape, Fund. Math. 127 (1987), 213-224.

[2] K. Borsuk, On movable compacta, Fund. Math. 66 (1969), 137-146.

[3] K. Borsuk, Theory of retracts, Monografie Matematyczne, Tom 44 Państwowe Wydawnictwo Naukowe, Warszawa, 1967.

[4] K. Borsuk, Theory of shape, Lecture Notes Series 28 Matematisk Institut, Aarhus Universitet, Aarhus, 1971.

[5] G.E. Bredon, Introduction to compact transformation groups, New York, 1972.

[6] Z. Čerin, Equivariant shape theory, Math. Proc. Camb. Phil. Soc. 117 (1995), 303320 .

[7] J. De Vries, Topics in the theory of topological transformation groups, Topological structures II, Mathematical centre tracts 116 (1979), 291-304.

[8] R. Engelking, General Topology, Polish Scientific Publishers, Warszawa, 1977.

[9] P.S. Gevorgyan, G-movability of G-spaces, Uspehi Mat. Nauk 43 (1988), 177-178 (in Russian).

[10] Hu Sze-Tsen, Homotopy Theory, Academic Press, New York, 1959.

[11] G. Kozlowski and J. Segal, Movability and shape-connectivity, Fund. Math. 93 (1976), 145-154.

[12] Yu.T. Lisica, Extension of continuous maps, Sibirski Matem. Ž. 14 (1973), 128-139 (in Russian).

[13] S. Mardešić, A non-movable compactum with movable suspension, Bull.Acad. Polon. Sci. 19 (1971), 1101-1103.

[14] S. Mardešić and J. Segal, Movable compacta and ANR-systems, Bull.Acad. Polon. Sci. 18 (1970), 649-654.

[15] S. Mardešić and J. Segal, Shape theory - The inverse system approach, North-Holland, Amsterdam, 1982.

[16] K. Morita, On shapes of topological spaces, Fund. Math. 86 (1975), 251-259.

[17] R.S. Palais, The classification of G-spaces, Mem. AMS 36 (1960). 
[18] Yu. M. Smirnov, Shape theory for G-pairs, Uspehi matem. nauk 40 (1985), 151-165 (in Russian).

Department of Higher Geometry and Topology

Moscow State University

Russia

E-mail: gevorgyan@mech.math.msu.su, pgev@angelfire.com

Received: 31.08 .2001

Revised: 22.01.2003. \& 26.05.2003 